\title{
La Cultura Financiera en las Microempresas Lima Este, año 2017
}

\author{
The Financial Culture in the Lima Este Micro-Enterprises, \\ year 2017
}

\section{RESUMEN}

Lima Este al 2014 cuenta con 172 481, microempresas (Cieneguilla 1 503, Chaclacayo 3 250, San Luis 9 144, Lurigancho 10 793, El Agustino 11 281, La Molina 16 500, Santa Anita 18 973, Ate 41874 y San Juan de Lurigancho 59 163), qué representa el $20 \%$ del total; concentra el $29.9 \%$ de la población total de Lima Metropolitana; según el Instituto Nacional de Estadística e Informática (INEI, 2015) uno de los factores principales que limitan el crecimiento de las empresas es la falta de accesibilidad al financiamiento de los productos financieros que ofrecen las entidades financieras. En ese sentido, los resultados de la encuesta realizado en los distritos que conforman el Lima Este en noviembre (2017), nos muestran los siguientes resultados: El 77.7\% de las Microempresas de Lima Este se encuentran en los distritos de San Juan de Lurigancho, Ate, La Molina y el Agustino; El 83.9\% se dedican a la actividad del Comercio y Servicios, el 71.2\% son sujetos de créditos, $71.8 \%$ conocen su endeudamiento y el $92 \%$ requieren créditos para el financiamiento del capital de trabajo y la compra de activo fijo.

Palabras claves: Cultura financiera; activo fijo; capital de trabajo; financiamiento.

\begin{abstract}
Lima East to 2014 has 172 481, micro businesses (Cieneguilla 1 503, Chaclacayo 3 250, San Luis 9 144, Lurigancho 10 793, El Agustino 11 281, La Molina 16 500, Santa Anita 18 973, Ate 41874 and San Juan de Lurigancho 59 163), which represents $20 \%$ of the total; it concentrates $29.9 \%$ of the total population of Metropolitan Lima. According to the INEI (2015) one of the main factors that limit the growth of companies is the lack of accessibility to the financing of financial products offered by financial institutions. By the way, the results of the survey conducted in the districts that make up the East Lima in November (2017), show us the following results:

$77.7 \%$ of the Micro Enterprises of Lima Este are located in the districts of San Juan de Lurigancho, Ate, La Molina and Agustino; $83.9 \%$ are dedicated to the activity of Commerce and Services, $71.2 \%$ are subject to loans, $71.8 \%$ know their indebtedness and $92 \%$ require loans for the financing of working capital and the purchase of fixed assets.
\end{abstract}

Keywords: Financial culture; fixed assets; working capital; financing.

\footnotetext{
${ }^{1}$ Doctor en Administración, Magíster en Administración con Mención en Gestión Empresarial, Docente asociado de pregrado y posgrado de la Facultad de Ciencias Administrativas de la Universidad Nacional Mayor de San Marcos.

(C) Los autores. Este artículo es publicado por Gestión en el Tercer Milenio de la Facultad de Ciencias Administrativas de la Universidad Nacional Mayor de San Marcos. Este es un artículo de acceso abierto, distribuido bajo los términos de la licencia Creative Commons Atribucion - No Comercia_Compartir Igual 4.0 Internacional. (http://creativecommons.org/licenses/by-nc-sa/4.0/) que permite el uso no comercial, distribución y reproducción en cualquier medio, siempre que la obra original sea debidamente citada.
} 


\section{INTRODUCCIÓN}

Según INEI (2014), existen 861 mil microempresas en Lima Metropolitana, de las cuales 172481 se encuentra en Lima Este (Cieneguilla 1 503, Chaclacayo 3 250, San Luis 9 144, Lurigancho 10 793, El Agustino 11 281, La Molina 16 500, Santa Anita 18 973, Ate 41874 y San Juan de Lurigancho 59 163), que representa el $20 \%$ del total; en términos de población, Lima Metropolitana tiene 8.7 millones de habitantes, Lima Este cuenta con 2.6 millones de habitantes qué representa el 29.9\% del total de Lima. La PEA de Lima Metropolitana asciende a 4.9 habitantes de las cuales $60 \%$ se encuentra en el empleo informal y $40 \%$ en el empleo formal.

Según el INEI (2015) Los factores principales que limitan el crecimiento de las empresas son la informalidad, acceso al financiamiento, la excesiva regulación tributaria y la competencia informal para su principal producto.

La accesibilidad al financiamiento en una entidad financiera, es muy importante para el crecimiento de las actividades de las microempresas, muchos inician sin tener conocimientos de los productos financieros que faciliten su desarrollo a lo largo de los años, obteniendo después graves problemas de administración, es importante cobrar conciencia sobre la administración el dinero, contar con un fondo de emergencias y usar de forma adecuada y responsable los productos y servicios financieros (Cruz, 2013). Tener una buena cultura financiera en las empresas, evita los endeudamientos, o el mezclar las finanzas personales con las finanzas de la empresa, ayudando a la obtención de más recursos, así como a una mejor vida financiera. A mayor cultura financiera por parte del empresario mayor será la demanda de los servicios financieros.

Por lo que se deduce como el problema: ¿De qué manera la cultura financiera incide al crecimiento de las actividades de las microempresas en Lima Este?

El objetivo de la presente investigación es el de determinar en qué medida la cultura financiera incidencia al crecimiento de las actividades de las microempresas en Lima Este.

\section{MÉTODO}

La investigación es de carácter aplicada, exploratoria, descriptivo, no experimental; la unidad de análisis es una microempresa; la población en estudio son 172481 microempresas; la muestra es 384 cuya selección fue probabilístico. Para la recopilación de la información se utilizado con instrumento el cuestionario y también, fuentes documentales del INEI.

\section{RESULTADOS}

Según la Encuesta de Micro y Pequeñas del INEI (2013), sobre el conocimiento de la micro y pequeña empresa sobre los productos financieros qué ofertaban las entidades financieras, el $94.5 \%$ se concentraron en créditos para capital de trabajo, seguido de créditos para inversión en activo fijo 48.9\%, seguros $13.3 \%$, ahorro $11.2 \%$, carta de fianza $11.1 \%$ y mientras productos como factoring y productos financieros estructurados están por debajo del $10 \%$. (Pregunta de respuesta múltiple).

En la encuesta realizado en los meses de noviembre del año 2017 en el ámbito de Lima Este con propósito cumplir con el objetivo de la investigación.

Del total encuestado el $32.5 \%$, se encuentran ubicados en el Distrito de San Juan de Lurigancho, 23.3\% en Ate, $11.3 \%$ en Santa Anita, $10.6 \%$ en La Molina, concentrándose en estos distritos el $77.7 \%$ del total de microempresas en el ámbito de la investigación (Tabla 1).

Según actividad económica, el 45.6\% se dedican al comercio, 38.3 a servicios, $9.8 \%$ a servicios y $2.1 \%$ a otros tipos de actividades (Tabla 2).

Los principales problemas qué las microempresas se enfrentan el ámbito de la investigación son de tipo comercial con un 39.4\%, seguido por la de económico financiero con $27.2 \%$, social con un $17.3 \%$, organizacional $14.4 \%$ y $1.7 \%$ otros tipos de problemas (Tabla 3 ).

Este resultado, es muy importante para la investigación, $71.8 \%$ de los encuestados manifiestan que son sujetos de crédito, esto significa que conocen los productos y servicios financieros que las entidades financieras los ofertan y el $28.2 \%$ no lo son. (Tabla 4). 
Tabla 1

Distritos del Cono Este al que pertenece su empresa

\begin{tabular}{|c|c|c|c|c|c|}
\hline Concepto & & Frecuencia & Porcentaje & Porcentaje válido & $\begin{array}{l}\text { Porcentaje } \\
\text { acumulado }\end{array}$ \\
\hline \multirow{10}{*}{ Distritos } & San Juan de Lurigancho & 125 & 32.5 & 32.5 & 32.5 \\
\hline & Ate & 89 & 23.3 & 23.3 & 55.8 \\
\hline & Santa Anita & 44 & 11.3 & 11.3 & 67.1 \\
\hline & La Molina & 41 & 10.6 & 10.6 & 77.7 \\
\hline & El Agustino & 26 & 6.8 & 6.8 & 84.5 \\
\hline & Lurigancho & 26 & 6.8 & 6.8 & 91.3 \\
\hline & San Luis & 22 & 5.7 & 5.7 & 97.0 \\
\hline & Chaclacayo & 8 & 2.1 & 2.1 & 99.1 \\
\hline & Cieneguilla & 4 & 0.9 & 0.9 & 100.0 \\
\hline & Total & 384 & 100.0 & 100.0 & \\
\hline
\end{tabular}

Fuente: Encuesta a Micro y Pequeñas Empresas noviembre 2017

Tabla 2

Sector Económico pertenece su empresa

\begin{tabular}{llcccc} 
Concepto & Frecuencia & Porcentaje & Porcentaje válido & $\begin{array}{c}\text { Porcentaje } \\
\text { acumulado }\end{array}$ \\
& Comercio & 175 & 45.6 & 45.6 & 49.8 \\
Sector Económico & Industria & 38 & 9.8 & 9.8 & 59.7 \\
& Servicios & 147 & 38.3 & 38.3 & 97.9 \\
& Otros & 8 & 2.1 & 2.1 & 100.0 \\
\hline
\end{tabular}

Fuente: Encuesta a Micro y Pequeñas Empresas noviembre 2017

Tabla 3

Los principales problemas que enfrenta la empresa

$\begin{array}{llcccc}\text { Concepto } & & \text { Frecuencia } & \text { Porcentaje } & \text { Porcentaje válido } & \begin{array}{c}\text { Porcentaje } \\ \text { acumulado }\end{array} \\ & \text { Comercial } & 151 & 39.4 & 39.4 & 39.4 \\ & \text { Social } & 66 & 17.3 & 17.3 & 56.7 \\ \text { Tipos de prob- } & \text { Económicos financieros } & 104 & 27.2 & 27.2 & 83.9 \\ \text { lemas } & \text { Organizacional } & 55 & 14.4 & 14.4 & 98.3 \\ & \text { Otros } & 7 & 1.7 & 1.7 & 100.0 \\ \end{array}$

Fuente: Encuesta a Micro y Pequeñas Empresas noviembre 2017

Tabla 4

Sujeto a crédito por las entidades financieras

\begin{tabular}{llcccc} 
Concepto & & Frecuencia & Porcentaje & Porcentaje válido & $\begin{array}{c}\text { Porcentaje } \\
\text { acumulado }\end{array}$ \\
\multirow{3}{*}{ Alternativa } & Sí & 276 & 71.8 & 71.8 & 71.8 \\
& No & 108 & 28.2 & 28.2 & 100.0 \\
& Total & 384 & 100.0 & 100.0 & \\
\hline
\end{tabular}

Fuente: Encuesta a Micro y Pequeñas Empresas noviembre 2017 
Tabla 5

Conocimiento del Endeudamiento de la empresa

$\begin{array}{llcccc}\text { Concepto } & & \text { Frecuencia } & \text { Porcentaje } & \text { Porcentaje válido } & \begin{array}{c}\text { Porcentaje } \\ \text { acumulado }\end{array} \\ & \text { Sí } & 273 & 71.2 & 71.2 & 71.2 \\ \text { Alternativa } & \text { No } & 111 & 28.8 & 28.8 & 100.0 \\ & \text { Total } & 384 & 100.0 & 100.0 & \end{array}$

Fuente: Encuesta a Micro y Pequeñas Empresas noviembre 2017

Tabla 6

Tipo de financiamiento qué necesitan

\begin{tabular}{llcccc} 
Concepto & Frecuencia & Porcentaje & $\begin{array}{c}\text { Porcentaje } \\
\text { válido }\end{array}$ & $\begin{array}{c}\text { Porcentaje } \\
\text { acumulado }\end{array}$ \\
& Capital de trabajo & 199 & 51.9 & 51.9 & 51.9 \\
Compra de activo fijo & 154 & 40.1 & 40.1 & 92.0 \\
Tinanciamiento de la & 27 & 7.0 & 7.0 & 98.9 \\
$\begin{array}{l}\text { Fipo de financiamiento } \\
\text { importación }\end{array}$ & Financiamiento de la & 3 & 0.8 & 0.8 & 99.7 \\
exportación & 1 & 0.3 & 0.3 & 100.0 \\
\hline
\end{tabular}

Fuente: Encuesta a Micro y Pequeñas Empresas noviembre 2017

Este cuadro, no hace más que confirmar, lo del párrafo anterior; el $71.2 \%$ de los encuestados manifiestan de qué si conocen el endeudamiento de sus empresas, mientras el $28.8 \%$ no los conocen. (Tabla 05 ).

Respecto al financiamiento requerido por los microempresarios; el $51.9 \%$ requieren créditos para el capital de trabajo, $40.1 \%$ para la compra de activos fijos y $8.1 \%$ para exportación e importación (Tabla 6).

\section{DISCUSIÓN}

Según la encuesta, el principal problema de los microempresarios, son de tipo comercial, es decir el mercado, esto se debe, a la competencia desleal por parte de las actividades informales, que hace de que los costos de producción sean altas y por ende los compromisos financieros se incrementen.

Por otro lado, identifican el producto financiero que necesitan y para que lo necesitan, es decir hay una toma de decisiones racional, por consiguiente, una cultura financiera que hace saludable la gestión en los microempresarios de Lima Este.

\section{CONCLUSIÓN}

El 77.7\% de los Microempresarios de Lima Este se encuentran en los distritos de San Juan de Lurigancho, Ate, La Molina y el Agustino.
El 83.9\% de los microempresarios encuestados, se dedican a la actividad del Comercio y Servicios, el $71.2 \%$ son sujetos de créditos, $71.8 \%$ conocen su endeudamiento y el $92 \%$ de los encuestados requieren créditos para el financiamiento del capital de trabajo y la compra de activo fijo.

\section{REFERENCIAS BIBLIOGRÁFICAS}

Aguilera, A., \& Puerto, D. P. (2012). Crecimiento empresarial basado en la Responsabilidad Social. Revista Científica Pensamiento y Gestión, 0(32).

Araujo, K. (2018). La cultura financiera de los empresarios y la Planeación empresarial en las pequeñas empresas del sector textil - confecciones ubicadas en el Emporio comercial de Gamarra, período 2017. Universidad de San Martin de Porres.

Cruz, A., (2013). Educación financiera indispensable para los empresarios.

Feijoo, A. (2016). Sistematización de iniciativas de educación financiera en el Perú. Lima, IEP; Proyecto Capital; IDRC-CDRI; Ford Fundación (Miscelánea, 39).

INEI (2013) Encuesta de Micro y Pequeña Empresa

INEI (2014) Una Mirada a Lima Metropolitana Setiembre 2014. 